\title{
THE GENERAL LOCAL FORM OF AN ANALYTIC MAPPING INTO THE SET OF IDEMPOTENT ELEMENTS OF A BANACH ALGEBRA
}

\author{
J.-PH. LABROUSSE
}

(Communicated by Palle E. T. Jorgensen)

Dedicated to the memory of Professor Jacques Morgenstern

\begin{abstract}
This paper gives a general formula which describes any analytical mapping of a suitably small open neighborhood $U$ in $\mathbb{C}$ into the set of idempotent elements of any complex Banach algebra $\mathbf{B}$ and an application of this formula to the case when $\mathbf{B}$ is a Calkin algebra.
\end{abstract}

Let $\mathbf{B}$ be a complex Banach algebra, for any $X, Y \in \mathbf{B}$ let $[X, Y]=X Y-$ $Y X$, let $U$ be an open subset of $\mathbb{C}$ (which we can assume without loss of generality to contain 0 ), let $F(z)$ be an analytic mapping of $U$ into $\mathbf{B}$ and let $P \in \mathbf{B}$ be idempotent $(P \neq 0)$. Then the main result of this paper is the following:

Theorem 1. If $[F(0), P]=0$ there exists an open neighborhood $V$ of 0 in $\mathbb{C}$ and two aralytic mappings $P(z), R(z)$ of $V$ in $\mathbf{B}$ such that:

(i) $P(0)=P$ and for all $z \in V, P(z)$ is idempotent,

(ii) for all $z \in V,[R(z), P]=0$,

(iii) for all $z \in V, F(z)=P(z)+R(z)$.

Moreover, in a small enough neighborhood of 0 , the pair of mappings $P(z), R(z)$ is uniquely determined by properties (i) to (iii).

Definition 2. Let $z \in \mathbb{C}$, with $0 \leq|z| \leq \frac{1}{4}$ and set $f(z)=\frac{1}{2}-\sqrt{\frac{1}{4}-z}$. If $n=1,2, \ldots$ define $c_{n}$ (the $n$th Catalan number, cf. [1], [3]) as follows:

$$
c_{n}=\frac{f^{(n)}(0)}{n !} \text {. }
$$

Clearly:

$c_{n}=\frac{(2 n-2) !}{n !(n-1) !}=[2 n-(2 n-1)] \frac{(2 n-2) !}{n !(n-1) !}=2 \frac{(2 n-2) !}{(n-1) !(n-1) !}-\frac{(2 n-1) !}{n !(n-1) !} \in \mathbb{N}$.

Lemma 3 (see [3] for a slightly weaker version of this lemma). Using the notation introduced above we have:

Received by the editors March 14, 1994 and, in revised form, May 23, 1994.

1991 Mathematics Subject Classification. Primary 47A56, 46H30.

(C) 1995 American Mathematical Society 
(i) The series $\sum_{j=1}^{\infty} c_{j} z^{j}$ is uniformly convergent to $f(z)$ in $0 \leq|z| \leq \frac{1}{4}$.

(ii) $\forall n \in \mathbb{N}^{*}, c_{n+1}=\sum_{j=1}^{n} c_{j} c_{n+1-j}$.

Proof. (i) Let us show first that

$$
\sum_{j=1}^{\infty} c_{j}\left(\frac{1}{4}\right)^{j} \leq \frac{1}{2}
$$

If $n=1,2, \ldots$ set $S_{n}=\sum_{j=1}^{n} c_{j}\left(\frac{1}{4}\right)^{j}$. Then:

$$
\begin{aligned}
S_{n+1} & +\left(\frac{1}{4}\right)^{(n+1)} \frac{(2 n+1) !}{n !(n+1) !} \\
& =S_{n}+\left(\frac{1}{4}\right)^{(n+1)}\left(\frac{(2 n) !}{n !(n+1) !}+\frac{(2 n+1) !}{n !(n+1) !}\right) \\
& =S_{n}+\left(\frac{1}{4}\right)^{(n+1)} \frac{(2 n+2)(2 n) !}{n !(n+1) !}=S_{n}+\left(\frac{1}{4}\right)^{n} \frac{(2 n-1) !}{n !(n-1) !}
\end{aligned}
$$

Hence $S_{n}+\left(\frac{1}{4}\right)^{n} \frac{(2 n-1) !}{n !(n-1) !}$ is independent of $n$ and since for $n=1$ its value is $\frac{c_{1}}{4}+\frac{1}{4}=\frac{1}{2}$, (1) follows at once, and this implies that for $0 \leq|z|<\frac{1}{4}$ the function represented by the series is analytic and coincides with $f(z)$. By continuity this still holds for $|z|=\frac{1}{4}$ so that (i) is established and (ii) is easily derived from the fact that $(f(z))^{2}=\frac{1}{4}+\frac{1}{4}-z-2\left(\frac{1}{2}\right) \sqrt{\frac{1}{4}-z}=f(z)-z$.

Lemma 4. Let $A \in \mathbf{B}$. Set $A_{1}=[A, P], A_{2}=\left[A_{1}, P\right]$. Then:

(a) $\left[A_{2}, P\right]=A_{1}$.

(b) $P A_{1}+A_{1} P=A_{1}$.

(c) $P A_{2}+A_{2} P=A_{2}$.

(d) $P$ commutes with $A_{1}^{2}$, with $A_{2}^{2}$ and with $A_{1} A_{2}$.

(e) $A_{1} A_{2}+A_{2} A_{1}=0$.

(f) $A_{1}^{2}+A_{2}^{2}=0$.

Proof. (a), (b) and (c) are established by direct computation from the fact that $A_{1}=A P-P A$ and that $A_{2}=A P-P A-2 P A P$.

(d) is an obvious consequence of (b) and (c).

To prove (e) notice that $A_{1} A_{2}+A_{2} A_{1}=A_{1}\left(A_{1} P-P A_{1}\right)+\left(A_{1} P-P A_{1}\right) A_{1}=$ $A_{1}^{2} P-P A_{1}^{2}=0$, using (d). Finally (f) follows from the fact that $A_{1}^{2}+A_{2}^{2}=$ $A_{1}^{2}+\left(A_{1} P-P A_{1}\right)^{2}=A_{1}^{2}+A_{1} P A_{1} P+P A_{1} P A_{1}-A_{1} P A_{1}-P A_{1}^{2} P=A_{1}^{2}-$ $(I-P) A_{1}^{2}-P A_{1}^{2}=0$.

Lemma 5. Let $A \in \mathbf{B}$ be such that $\left\|A_{1}\right\| \leq 1 /(4\|P\|)$. Then

$$
Q=P+A_{2}+\sum_{j=1}^{\infty} c_{j} A_{1} A_{2}^{2 j-1} \quad \text { is an idempotent element of } \mathbf{B} .
$$

Proof. Under the given hypothesis $\left\|A_{2}\right\| \leq 2\left\|A_{1}\right\|\|P\| \leq \frac{1}{2}$. Hence using 
Lemma 3, the series that defines $Q$ is uniformly convergent. Furthermore:

$$
\begin{aligned}
Q^{2}= & P+P A_{2}+\sum_{j=1}^{\infty} c_{j} P A_{1} A_{2}^{2 j-1}+A_{2} P+A_{2}^{2}+\sum_{j=1}^{\infty} c_{j} A_{2} A_{1} A_{2}^{2 j-1} \\
& +\sum_{j=1}^{\infty} c_{j} A_{1} A_{2}^{2 j-1} P+\sum_{j=1}^{\infty} c_{j} A_{1} A_{2}^{2 j}+\sum_{j=1}^{\infty} \sum_{k=1}^{\infty} c_{j} c_{k} A_{1} A_{2}^{2 j-1} A_{1} A_{2}^{2 k-1} \\
= & P+A_{2}+2 \sum_{j=1}^{\infty} c_{j} P A_{1} A_{2}^{2 j-1}+A_{2}^{2} \\
& +\sum_{j=1}^{\infty} \sum_{k=1}^{\infty} c_{j} c_{k} A_{1} A_{2}^{2 j-1} A_{1} A_{2}^{2 k-1} \quad \text { (using (b), (c) and (e)) } \\
= & P+A_{2}+2 P A_{1} \sum_{j=1}^{\infty} c_{j} A_{2}^{2 j-1}+A_{2}^{2}-\sum_{i=1}^{\infty} \sum_{k=1}^{\infty} c_{i+1-k} c_{k} A_{1}^{2} A_{2}^{2 i} \\
= & P+A_{2}+\left(A_{1}-A_{2}\right) \sum_{j=1}^{\infty} c_{j} A_{2}^{2 j-1}+A_{2}^{2} \\
& +\sum_{i=1}^{\infty} c_{i+1} A_{2}^{2 i+2} \quad(\text { using (f) and Lemma 3) } \\
= & P+A_{2}+\sum_{j=1}^{\infty} c_{j} A_{1} A_{2}^{2 j-1}=Q .
\end{aligned}
$$

We are now in a position to prove Theorem 1.

Existence of the decomposition. Let $F(z)$ be analytic mapping of $U$ into B such that $[F(0), P]=0$. Then there exists a neighborhood $V \subseteq U$ such that $\forall z \in V,\|[F(z), P]\|<1 /(4\|P\|)$ and therefore the mapping of $V$ into $B$ defined by

$$
P(z)=P+[[F(z), P], P]+\sum_{j=1}^{\infty} c_{j}[F(z), P][[F(z), P], P]^{2 j-1}
$$

is analytic on $V$. Note that $P(0)=P$ and that $\forall z \in V, P(z)$ is idempotent. Then, if $R(z)=F(z)-P(z)$,

$$
\begin{aligned}
{[R(z), P]=} & {[F(z), P]-[P(z), P] } \\
= & {[F(z), P]-[F(z), P] } \\
& +\sum_{j=1}^{\infty} c_{j}\left\{[F(z), P][[F(z), P], P]^{2 j-1} P\right. \\
= & \left.0 \quad-P[F(z), P][[F(z), P], P]^{2 j-1}\right\}
\end{aligned}
$$

using Lemma 4. Hence the existence of the decomposition is proved.

Uniqueness of the decomposition. Assume that $F(z)=P_{1}(z)+R_{1}(z)=P_{2}(z)+$ $R_{2}(z)$ with $P_{1}(0)=P_{2}(0)=P$. Set $D(z)=P_{1}(z)-P_{2}(z)=R_{1}(z)-R_{2}(z)$. 
Then $D(z)$ commutes with $P$ and $D(z)=P_{1}^{2}(z)-P_{2}^{2}(z)=D(z) P_{1}(z)+$ $P_{2}(z) D(z)$. Let us show by induction that

$$
\forall n \in \mathbb{N}, \quad D^{(n)}(0)=0 .
$$

For $n=0$ we see that $D(0)=P_{1}(0)-P_{2}(0)=0$. Assume that (3) has been proved for $n=0,1, \ldots, r$. Then using Leibniz's formula we get

$$
\begin{aligned}
D^{(r+1)}(0) & =\sum_{j=0}^{r+1}\left(\begin{array}{c}
r+1 \\
j
\end{array}\right)\left\{D^{(r+1-j)}(0) P_{1}^{(j)}(0)+P_{2}^{(j)}(0) D^{(r+1-j)}(0)\right\} \\
& =D^{(r+1)}(0) P+P D^{(r+1)}(0),
\end{aligned}
$$

using the induction hypothesis. So $D^{(r+1)}(0)=2 P D^{(r+1)}(0)$, whence $P D^{(r+1)}(0)$ $=2 P D^{(r+1)}(0)$ so that $P D^{(r+1)}(0)=0$, therefore $D^{(r+1)}(0)=0$ and $(3)$ is proved. Hence $D(z)=0$, which concludes the proof of the theorem.

Remark. (2) gives the general local form of any analytic mapping of a neighborhood of 0 into the set of idempotent elements of $\mathbf{B}$. Indeed suppose that $P$ is such a mapping defined on a neighborhood $U$ of 0 into $\mathbf{B}$ and set $P=P(0)$. Then $P(0) P=P P(0)$ and by Theorem 1 there exists a neighborhood $V$ of $\{0\}$ such that

$$
Q(z)=P+[[P(z), P], P]+\sum_{j=1}^{\infty} c_{j}[P(z), P][[P(z), P], P]^{2 j-1}
$$

is an analytical mapping of a neighborhood $V$ of 0 into the set of the idempotent elements of $\mathbf{B}$ and we have

$$
P(z)=Q(z)+R(z)=P(z)+0
$$

and since the decomposition is unique, it follows that $Q(z)=P(z)$ and consequently that $P(z)$ is locally of the form given by (2).

Application. Let $H$ be a complex Hilbert space, and let $L(H)$ denote the algebra of all bounded linear operators on $H$ and $K(H)$ the closed bilateral ideal constituted by the compact operators of $L(H)$. Then $A(H)=L(H) / K(H)$ is the Calkin algebra associated to $L(H)$. Let $\pi$ denote the natural mapping of $L(H)$ onto $A(H)$.

Theorem 2. Let $U$ be a neighborhood of 0 in $\mathbb{C}$, and let $p(z)$ be an analytic mapping of $U$ into the space of the idempotent elements of $A(H)$. Then there exists a neighborhood $V$ of 0 in $\mathbb{C}$ and an analytic mapping $P(z)$ of $V$ into the set of the idempotent elements of $L(H)$ such that

$$
\forall z \in V, \quad p(z)=\pi(P(z)) .
$$

Proof. By assumption $\forall z \in U, p(z)=\sum_{j=0}^{\infty} p_{j} z^{j}$ where $p_{0}$ is idempotent.

According to [2], Proposition 7, there exists $F_{0} \in L(H)$, idempotent, such that $\pi\left(F_{0}\right)=p_{0}$. Furthermore for every $j \geq 1$ there exists $F_{j} \in L(H)$ such that $\pi\left(F_{j}\right)=p_{j}$ with $\left\|F_{j}\right\| \leq 2\left\|p_{j}\right\|$ (this is an obvious consequence of the definition of the norm in $A(H)$ ). Hence $F(z)=\sum_{j=0}^{\infty} F_{j} z^{j}$ converges and is analytic in some neighborhood $V$ of 0 and we have $\forall z \in V, \pi(F(z))=$ $p(z)$. But $F(0)=F_{0}$. So using Theorem 1, taking $P=F_{0}, F(z)=P(z)+$ 
$R(z)$, and therefore $p(z)=\pi(P(z))+\pi(R(z))$ where $\pi(P(z))$ is idempotent with $\pi(P(0))=p_{0}$ and $\pi(R(z))$ commutes with $p_{0}$. Hence, because of the uniqueness of the decomposition, $\pi(R(z))=0$ and we have shown that there exists an idempotent-valued mapping $P(z)$, analytic on $V$, such that

$$
\forall z \in V, \quad p(z)=\pi(P(z)) .
$$

\section{REFERENCES}

1. E. Catalan, Note sur une équation aux différences finies, J. Math. Pure Appl. 3 (1838), 508-517.

2. P. De La Harpe, Initiation à l'algèbre de Calkin, Lecture Notes in Math, vol. 725, SpringerVerlag, Berlin and New York, 1979, pp. 180-219.

3. J. H. van Lint and R. M. Wilson, A course in combinatorics, Cambridge Univ. Press, London and New York, 1992, pp. 116-117.

Laboratoire J. A. Dieudonné, URA CNRS 168, Université de Nice Sophia Antipolis, Parc Valrose, 06108 Nice Cedex 2, France

E-mail address: labro@math.unice.fr 